\title{
THE CARE OF RHEUMATIC CHILDREN
}

\section{REPORT BY THE CARDIAC SOCIETY AND BRITISH PAEDIATRIC ASSOCIATION}

In 1942 the Cardiac Society appointed a small committee to draw up a report and make suggestions about the arrangements that should be made for the care of rheumatic children. When it was learnt that the British Paediatric Association had appointed a similar committee and that some members were serving on both bodies, it was hoped that a joint report might be issued. This wish was shared by the British Paediatric Association and the two committees met again as a joint committee and drew up a report. After various minor modifications, the Council of the Cardiac Society and the Executive of the British Paediatric Association agreed to publish the report that follows. They are greatly indebted to the following members who drew up the original joint report:

F. M. B. Allen (B.P.A.), J. V. Braithwaite (B.P.A.), T. F. Cotton (C.S.), W. Evans (C.S.), C. B. Perry (B.P.A. and C.S.), C. T. Potter (B.P.A.), J. C. Spence (B.P.A.), A. G. Watkins (B.P.A.), K. D. Wilkinson (B.P.A. and C.S.), T. P. Williams (B.P.A.), and B. Schlesinger (C.S.) (who was prevented from agreeing to the final draft owing to absence on active service).

Acute rheumatism is primarily a diseasc of school age and often produces serious results. It impairs physical health, causes serious loss of education by preventing attendance at school, is the main cause of organic cardiac disease in early life, and may lead to physical incapacity for years and to death relatively early in adult life.

Until it is possible to prevent children becoming the victims of this disease, treatment must be limited to early diagnosis and the use of those measures which are known to hold out the best prospects of limiting the cardiac damage.

These objects can best be effected by the following:-

1. The establishment of cardio-rheumatic clinics where the diagnosis can be established early and with certainty.

2. The organization of hospital schools where children can be efficiently treated for so long as may be necessary while education continues.

3. The compulsory notification of all cases of acute rheumatism, chorea and rheumatic heart disease.

These steps may ultimately prove desirable for the whole country. At the moment, however, it is suggested that a trial should be made in certain selected centres (e.g. large towns with medical schools) where suitable personnel for staffing clinics and hospital schools would be available. It must be stressed that these three actions must be taken concurrently and that there would be no object in setting up notification if no clinic were established or in starting a clinic unless it has access to a hospital school.

The cardio-rheumatic clinic.-This should be situated in such a position that it is easy of access from the surrounding district. It should be closely associated with a key hospital, so that good laboratory facilities may be readily available, and the clinical material of the clinic may be utilized for teaching purposes. It should be purely consultative and advisory, with the objects of diagnosis and supervision, of follow-up, of research, and possibly of the direction of adolescent patients into suitable occupations.

Consultations should be by appointment (where necessary transport should be provided), and school medical officers and general practitioners should be encouraged to attend. The clinic should be held at least once a week, and when needed an evening session should be arranged to meet the requirements of patients in employment. The follow-up and supervision should continue into adult life. This clinic should be staffed by physicians with experience of children's diseases and diseases of the heart. This is essential to enable the clinic to deal with problems arising in the differential diagnosis of rheumatic manifestations and to follow up cardiac cases into adult life. In some centres it may be possible to find one physician who combines these functions, but in others it will be necessary to make use of a team. They should have the help of an assistant physician or registrar. An Almoner should be attached to the clinic and adequate secretarial assistance must also be provided.

It is desirable that the equipment should include: apparatus for measuring height and weight; an $\mathrm{x}$-ray set for screening and photographing hearts; an electrocardiograph and a technician; a laboratory for sedimentation rates, blood counts and biochemical investigations:

The function of this clinic should be the early diagnosis of acute rheumatism in children and adolescents, and the follow-up of those patients who have had carditis, with the following objects:-

1. Securing the best possible treatment so as to minimize cardiac damage.

2. Supervising the life and activities of the rheumatic child. 
3. The compilation of reliable data with the object of securing prevention of acute rheumatism.

4. The direction of cardiac defectives into suitable occupations.

5. The education of medical practitioners in the diagnosis of acute rheumatic carditis and cardiac disease generally.

6. The differentiation of habit spasms and tics from chorea, and 'growing pains' from acute rheumatism, which experience has shown form a large part of the work of such a clinic.

Consultations at the homes of children should be made possible. The parents, the family doctor, and the school medical officer should be advised as to fitness for school and any treatment needed. A report should be sent to the family doctor and school medical officer.

All cases of acute rheumatism, chorea and carditis notified should be immediately referred to the clinics unless admitted to the hospital school. School medical officers and general practitioners should be authorized and encouraged to refer to the clinic all children under the age of 16 in whom cardiac murmurs of doubtful significance are found. This is important for two reasons:-

(a) There is often difficulty in deciding whether a given murmur is ${ }^{*}$ evidence of past or present carditis or a congenital malformation, or is an 'innocent' or 'functional' murmur; it is most important that any such doubt should be removed so that unnecessary restrictions need not be imposed.

(b) The proper examination of such cases has a great value from the point of view of research and education.

It is desirable in addition that all children with a history of past rheumatism or chorea, even though they have no signs of cardiac damage, should also be referred to the clinic so that they may be kept under supervision and any relapse noted as soon as possible.

In addition to the report and advice on school and treatment a note should be made as to the necessity for another examination (i.e. in 6 weeks, 3 months, 6 months, etc.), and arrangements made for the child to be summoned to the clinic again at that time.

Hospital schools.-Such hospitals should be established at suitable points throughout the country. They should be hospitals for treatment of children with cardiac rheumatism, but should also be equipped with well qualified teachers and full school apparatus so that educational facilities may be available for all those inmates who can benefit by education. In addition to continuing general education, each child should be trained as far as may be possible so that he is able to earn a living without the necessity of laborious physical work. By various grades of recreational education both knowledge and manual skill may be acquired.

Where possible these schools should be situated in the country, and they should be large enough to accommodate all cases of acute rheumatism arising in the district served. The stay of each child should be a period of many months. Each hospital school must be supplied with adequate hospital equipment so that nursing of severely ill children can be carried out and so that progress may be recorded-i.e. an electrocardiograph and a technician, an x-ray screen and a suitable laboratory. Each should be a centre for research into etiological factors and improved treatment of acute rheumatism. In these hospital schools the treatment of acute rheumatism might well be combined with that of other 'long stay' children's diseases. This may be particularly necessary in view of the probable shortage of skilled teachers of the necessary type since it is clearly desirable that all children suffering-from long illnesses should be provided with educational facilities.

Where possible the physician in charge of the cardio-rheumatic clinic should also visit and supervise the hospital school and the assistant physician or registrar should also be attached to the hospital school.

Children will be admitted to the hospital school either direct from their homes immediately on notification, from other hospitals, or from the clinic, and patients should be retained in the hospital school until either (a) their condition is proved not to be due to acute rheumatism or other cause of cardiac damage, or (b) it is certain that the infection is quiescent or the condition cured, and the child is fit to live at home. On return they should, whenever possible, attend ordinary schools. Experience has shown that unless there is considerable cardiac enlargement these children are well able to lead a normal school life with full activities. Cases with more severe cardiac damage can attend ordinary schools provided they do no competitive games or drill. P.D. schools of a special type may be of great value by enabling education to continue until the age of 16 and by providing a special vocational training in the final years. But it must be remembered that it is most desirable from the psychological point of view that these children should be brought up as much like normal children as possible. A small number of children may be so severely damaged that it is advisable to transfer them to an institution for chronic invalids if they cannot be adequately cared for at home. Immediately on discharge from the hospital school the child should be referred back to the clinic for supervision.

In view of the striking social incidence of acute rheumatism every effort should be made to promote satisfactory housing for all children and to see that all children receive adequate diet and clothing. Children who have had acute rheumatism should be given special home visits with supervision, and improvement of home environment whenever possible.

The public and the profession should be educated to realize the potential importance of upper respiratory infection, however trivial it may appear. Parents of children attending the supervisory clinics should be instructed to report any such infection at 
once so that proper care may be instituted in an attempt to prevent a rheumatic relapse.

\section{Other measures}

Vocational training.-Facilities must be made available for vocational training of rheumatic children during their last years at school and on leaving school so that they are trained for suitable sedentary employment. When this has been done steps must be taken to ensure that when they start work they do in fact obtain such employment.

Notification.-The serious results of rheumatic carditis and the importance of early diagnosis and adequate treatment make it imperative that all cases of suspected acute rheumatism, chorea and rheumatic heart disease in children under the age of sixteen years should be made compulsorily notifiable.

It should be the duty of any medical practitioner who suspects that a child or young person is suffering from any of the above-named conditions to notify immediately the local Health Authority.

It should be the duty of the local Health Authority upon the receipt of such notification to refer the child to a cardio-rheumatic clinic and if necessary to offer to the parents accommodation for the child in a hospital school. It should be possible for the notification to be cancelled if, after examination at the clinic, it is found that the child is not suffering from rheumatism, chorea or rheumatic heart disease.

Research.-All clinics and hospital schools should be so staffed and equipped that they become active centres of research. This research should be coordinated by frequent interchange of ideas amongst the staffs of the various regions. This could perhaps be best facilitated by a co-ordinating committee who should be informed of all research in progress. 